\title{
Efficacy of mindfulness-based meditation therapy on the sense of coherence and mental health of nurses
}

\author{
Michiyo Ando ${ }^{1^{*}}$, Takako Natsume ${ }^{2}$, Hiroko Kukihara ${ }^{1}$, Hiroko Shibata ${ }^{3}$, Sayoko Ito ${ }^{4}$ \\ ${ }^{1}$ St. Mary’s College, Fukuoka, Japan; *Corresponding Author: andou@st-mary.ac.jp \\ ${ }^{2}$ Yame General Hospital, Fukuoka, Japan \\ ${ }^{3}$ St. Mary’s Hospital, Fukuoka, Japan \\ ${ }^{4}$ Kyoto Seizan Junior College, Kyoto, Japan
}

Received 2 December 2010; revised 1 February 2011; accepted 15 February 2011.

\section{ABSTRACT}

The primary goal of the study was to assess the efficacy of mindfulness-based meditation therapy on the psychological well-being and sense of coherence of nurses with respect to coping with stress. The participants were 28 nurses working in a ward for elderly patients, with 15 included in the intervention group and 13 in the control group. Nurses in the intervention group received two sessions of mindfulness-based meditation therapy, including breathing, yoga movement and meditation. Each nurse was taught the program in the first session, then exercised at home with a CD, and subsequently met with an interviewer in a second session after two weeks. Nurses in the control group did not receive any interventions. The participants completed the General Health Questionnaire (GHQ), including subdomains of physical symptoms, anxiety and sleep disturbance, interference with social activities, and depression; and the Sense of Coherence (SOC) questionnaire, which includes three subdomains of comprehensibility, manageability, and meaningfulness pre- and post-intervention. The GHQ scores of the intervention group showed a significantly greater decrease than those of the control group. The SOC scores of the intervention group showed a significantly greater increase after the intervention compared to those in the control group, with the highest score for the meaningfulness subdomain. These results indicate that mindfulness-based meditation therapy is effective for improving the psychological well-being and sense of coherence of nurses, which helps them to cope with stress.
Keywords: Meditation Therapy;

Sense of Coherence; Well-Being; Stress; Nurses

\section{INTRODUCTION}

Nurses tend to feel high levels of stress that may be caused by multiple factors [1-3]. The concept of sense of coherence for coping with stress was introduced by Antonovsky [4] and includes key factors that affect recovery from illness and maintenance of health. The sense of coherence consists of three factors: a sense of comprehensibility, through which a person can comprehend a stressful event; a sense of manageability, through which a person can manage a stressful event; and a sense of meaningfulness, through which a person feels meaning in a stressful event [5]. Previous studies have shown an association of the sense of coherence (SOC) score and other variables. A high SOC score is related to a low feeling of fatigue [6], low burnout [7], and a low mental or physical stress response [8]. For nurses, a high SOC is related to low burnout $[9,10]$ and high work satisfaction. These studies suggest the importance of SOC for coping with stress.

A mindfulness approach is an effective intervention in stress management from a psychological perspective. The Mindfulness-Based Stress Reduction (MBSR) program is modeled on the work of Kabat-Zinn and colleagues at the Center for Mindfulness at the Massachusetts Medical Center [11]. The program is based on the principle of mindfulness, defined as moment-to-moment, present-centered, purposive non-judgmental awareness. The goal of the MBSR program is to guide participants to achieve greater awareness of themselves, their thoughts, and their bodies through class discussion, meditation, and yoga exercises. It is effective for mood disturbance and stress symptoms [12] and for QOL and the immune profile [13]. Other studies have shown the effects of MBSR on symptoms of distress and health-related QOL 
[14] and spirituality [15]. However, few studies have examined interventions for stress management in nurses. Thus, the present study was performed to investigate the efficacy of mindfulness therapy on the sense of coherence and psychological well-being of nurses. The study was performed for nurses who work in wards for the elderly. These nurses may be under particular stress since many care workers have recently begun to work in these wards and the number of nurses has decreased.

\section{METHODS}

\subsection{Participants}

The participants were 28 nurses who worked in wards for elderly patients. Nurses in the intervention group ( $\mathrm{n}=$ 15) received mindfulness-based therapy, while those in the control group $(n=13)$ received no intervention. The inclusion criterion was age $>20$ years old, and the exclusion criterion was the presence of severe mental problems. The head nurse in the ward selected potential participants and explained the study to the nurses. All the subjects voluntarily participated in the study.

\subsection{Outcome Measures}

The Japanese short version of the SOC questionnaire [16] translated from the original version [5] was used in the study. The SOC scale has three subdomains that correspond to the sense of comprehensibility, sense of manageability, and sense of meaningfulness. The short version consists of 13 items, each of which is scored on a 7-point Likert scale. The General Health Questionnaire (GHQ) has 4 subdomains corresponding to physical symptoms, anxiety and sleep disturbance, interference with social activities, and depression. We used the Japanese version of GHQ 28[17]. The nurses also provided written information on stressful issues and commented on the effects of mindfulness therapy. The FACIT-Sp $[18,19]$ was used to measure spiritual well-being.

\subsection{Interventions and Procedure}

A modified version of the MBSR Program, in which we refer to cyclic meditation as that including both breathing and meditation, was used in the study. During meditation, nurses moved their hands or legs to focus their attention on mind and body. The cyclic meditation program takes 30 to 60 minutes per session and was conducted by nurses who received training in this program for at least 3 hours. The training included basic communication skills and Yoga skills learned directly from a Yoga specialist or using a CD or DVD. The subjects gave informed consent and completed pre- and post-intervention questionnaires. In the pre-therapy ses- sion, the subject learned the cyclic meditation program and it was recommended that the therapy was performed at home once a day. After 2 weeks, each subject met with an interviewer in a second session to talk about their impressions.

\subsection{Statistical Analysis}

A p value less than 0.05 was taken to indicate a significant level in all statistical analyses. All reported $\mathrm{p}$ values are 2-tailed. The statistical procedures were conducted with SPSS ver. 18.0 (Japanese version) for Windows (SPSS Inc.). A two-way repeated measures ANOVA [2 (groups: intervention, control) $\times 2$ (period: pre, post)] was used to evaluate the efficacy of mindfulness therapy for improving GHQ and SOC scores. A t-test was performed to compare the scores for the total GHQ and the GHQ subdomains for the intervention group preand post-intervention. Two-way ANOVA was performed for 2 periods (pre and post) $\times 3$ factors (comprehensibility, manageability, meaningfulness) to evaluate the efficacy of mindfulness therapy on the SOC subdomains. The free description of stress and the comments on mindfulness therapy given by the nurses were evaluated using content analysis [18].

\section{RESULTS}

\subsection{General Health Questionnaire (GHQ)}

Regarding the GHQ (Figure 1), the main effect of period was significant $(p=0.00)$ indicating that GHQ scores significantly decreased post-intervention. The main effect of the group was not significant. The interaction was also significant at a $10 \%$ level $(\mathrm{p}=0.059)$. The total GHQ scores for the intervention group significantly decreased from $11.13 \pm 4.72$ to $5.93 \pm 3.94$, indicating an improvement in general health, whereas those for the control group did not decrease. The subdomain scores for the intervention group decreased from $3.93 \pm 1.94$ to $2.20 \pm 1.97$ for physical symptoms, from $4.33 \pm 2.02$ to $2.87 \pm 2.00$ for anxiety and sleep disturbance, from $1.87 \pm$ 1.77 to $0.53 \pm 0.64$ for interference with social activities, and from $1.00 \pm 1.20$ to $0.33 \pm 0.62$ for depression (Table 1).

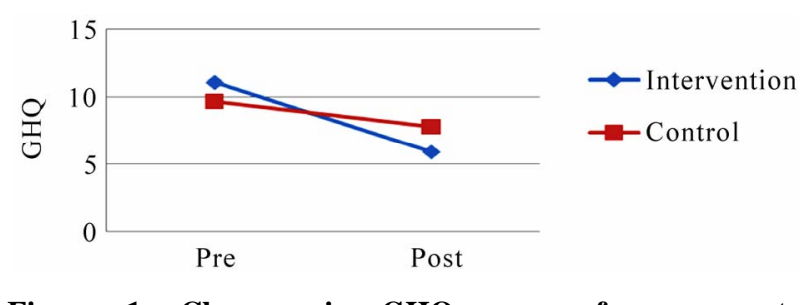

Figure 1. Changes in GHQ scores from pre- to post-intervention. 
Table 1. Pre- and post-intervention scores for the total GHQ and subdomains of the GHQ.

\begin{tabular}{cccccc}
\hline & Total GHQ & Physical symptoms & Anxiety, sleep & Social activities & Depression \\
\hline Pre & $11.1 \pm 4.72$ & $3.93 \pm 1.94$ & $4.33 \pm 2.02$ & $1.87 \pm 1.77$ & $1.0 \pm 1.20$ \\
Post & $5.9 \pm 3.94$ & $2.20 \pm 1.97$ & $2.87 \pm 2.00$ & $0.53 \pm 0.64$ & $0.33 \pm 0.62$ \\
$t$ & 6.5 & 5.03 & 3.08 & 3.00 & 2.47 \\
$p$ & 0.000 & 0.000 & 0.008 & 0.009 & 0.027 \\
\hline
\end{tabular}

\subsection{Sense of Coherence (SOC)}

For SOC (Figure 2), the main effect was not significant, but the interaction was significant $(\mathrm{p}<0.04)$. The SOC score for the intervention group significantly increased after the intervention, whereas that for the control group did not change. The score for the SOC meaningfulness subdomain significantly increased $(\mathrm{F}(1,14)=$ 7.94, $\mathrm{p}=0.014$ ), and the score for meaningfulness was higher than those for comprehensibility and manageability (Table 2).

\subsection{FACIT-Sp}

For the FACIT-Sp, the main effects and interaction were not significant. The FACIT-Sp score for the intervention group increased from $15.5 \pm 4.4$ to $16.6 \pm$, and that for the control group increased from $13.8 \pm 3.7$ to $13.6 \pm 3.1$.

\subsection{Comments}

Comments on stress and the effects of mindfulness therapy given by the subjects are shown in Table 3 . Many nurses indicated that a "labor shortage" and "overtime work" caused them to have stress. They found the mindfulness therapy to be relaxing and refreshing, and to give them time for reflection.

\section{DISCUSSION}

The greater decrease in GHQ scores in the intervention group compared to the control group indicates the efficacy of mindfulness therapy for the psychological well-being of nurses. Moreover, the scores for each subdomain significantly decreased after the intervention, which indicates the effectiveness of mindfulness therapy for physical symptoms, anxiety and sleep disturbance, interference with social activities, and depression. This result is consistent with those found for the effects of this therapy on anxiety [19,20] and mood [15]. The effect of the mindfulness-based meditation therapy is particularly interesting since the total GHQ score was 11.1 pre-intervention and the cutoff point for stress is $14 / 28$, indicating that the nurses in the present study did not have particular mental problems. However, the therapy

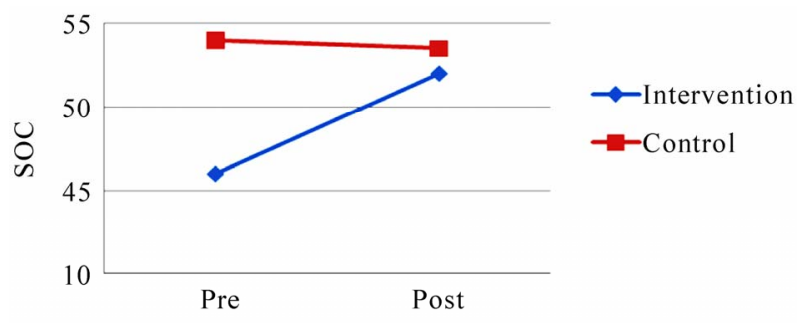

Figure 2. Changes in SOC scores from pre- to post-intervention.

Table 2. Pre- and post-intervention scores for sense of coherence (SOC) subdomains

\begin{tabular}{cccc}
\hline & Comprehensibility & Manageability & Meaningfulness \\
\hline Pre & $3.44 \pm 0.65$ & $3.35 \pm 0.77$ & $4.08 \pm 0.93$ \\
Post & $3.83 \pm 0.79$ & $3.70 \pm 0.82$ & $4.52 \pm 0.80$ \\
$t$ value & -2.06 & -1.62 & -2.30 \\
$p$ value & 0.058 & 0.128 & 0.037 \\
\hline
\end{tabular}

Table 3. Descriptions of stressful issues and comments on mindfulness therapy.

\section{Stress}

1) Lack of skills and knowledge about nursing

2) Overtime work

3) No payment for overtime work

4) Labor shortage

5) Unable to concentrate on my work

6) Human relationships with my nursing superior

7) Human relationships with doctors

8) Unable to care for patients or families because of too much work

Comments on mindfulness therapy

1) Useful for relaxation

2) Refreshing

3) Useful for reflection on my daily life

4) I found it difficult to focus my attention

5) It was difficult to make time for the mindfulness program

6) Yoga movements were not suitable for me 
was still effective in improving the psychological wellbeing of the nurses.

There was a greater increase in SOC scores in the intervention group than in the control group, which indicates the efficacy of mindfulness therapy on the sense of coherence in stress management. The standard SOC score is 54-58, while the scores of the nurses in the present study were 53 in the control group and 52 in the intervention group, indicating that the nurses had a poor ability to cope with stress. Thus, the results indicate that mindfulness therapy is important in facilitating stress management.

The experience level of nurses might influence the effects of mindfulness meditation therapy. Furthermore, the duration of the CD or DVD for this therapy was about 20 minutes, but some nurses took much more time to practice this therapy. The depth of meditation and focus of attention on the therapy might also have differed among the nurses. A further study including assessment of the experience of the subjects and the depth of focus during therapy will clarify the effects of these issues on the therapy.

The score for meaningfulness was the highest among the three SOC subdomains of comprehensibility, manageability, and meaningfulness. This suggests that the nurses were able to reflect on the meaning of their life and work through meditation. They were typically very busy and burdened by their work, and meditation allowed them to focus their attention on mind and body and to reflect on their purpose. In a previous intervention study, assertive training was show to affect the self- esteem of nurses, but had no effect on SOC [21]. This difference may be due to the type of intervention, since assertion reflects a positive attitude toward others, but mindfulness produces an accepting attitude toward others. Comprehensibility and manageability did not increase after the mindfulness therapy because nurses reported stress with their workload and the small number of nurses in the ward.

Cyclic meditation through mindfulness had no effect on spirituality in the present study. The standard FACIT-Sp score for meaning is 32, whereas the mean scores were 16 in the intervention group and 13.6 in the control group. This suggests that the nurses in the study may have lost a sense of meaning of life or purpose and peace of mind, and a further intervention to improve spirituality may be needed.

The study is limited by the small number of participants. To increase the robustness and reliability of the findings, a further study in more subjects is required. Moreover, we did not investigate the long-term effects of cyclic meditation therapy, and there is a need to test whether there is still a difference between GHQ and
SOC scores in the intervention and control groups after a longer period of time.

\section{ACKNOWLEDGEMENTS}

This research was supported by a Grant-in-Aid for Scientific Research (C).

\section{REFERENCES}

[1] Tarran, T. and Sabo, C.E. (2010) Role conflict, role ambiguity, and job satisfaction in nurse executives. Nursing Administration Quarterly, 34, 72-82.

[2] Innstrand, S.T., Langballe, E.M. and Falkum, E. (2010). Exploring occupational differences in work-family interaction: who is at risk? International Journal of Stress Management, 17, 38-55. doi:10.1037/a0018565

[3] Shirey, M.R., McDaniel, A.M. and Ebright. P.R. (2010). Understanding nurse manager stress and work complexity: factors that make a difference. The Journal of Nursing Administration, 40, 82-91. doi:10.1097/NNA.0b013e3181cb9f88

[4] Antonovsky, A. (1979). Health, stress, and coping: New perspective on mental and physical well-being. Jossey-Bass, San Francisco.

[5] Antonovsky, A. (1987). Unraveling the mystery of health: How people manage stress and stay well. Jossey-Bass, San Francisco.

[6] Hanse, J.J. and Engstrom T. (1999). Sense of coherence and ill health among the unemployed and reemployed after closure of assembly plant. Work and Stress, 13, 204222. doi:10.1080/026783799296020

[7] Gilver, O. (1988) Relationships between burnout and sense of coherence in health workers. Social Work in Health Care, 26, 39-49.

[8] Albertse, K., Nielsen, M.L. and Borg, V. (2001). The Danish psychosocial work environment and symptoms of stress. The main, mediating and moderating role of sense of coherence. Work and Stress, 15, 241-253. doi:10.1080/02678370110066562

[9] Tselebis, A., Moulou, A and Ilias, I. (2001). Burnout and sense of coherence: study of Greek nursing staff. Nursing \& Health Science, 3, 69-71. doi:10.1046/j.1442-2018.2001.00074.X

[10] Eda, S., Tatsumi, Y. and Nomura, M. (2007). The relationships between sense of coherence and burnout of critical care nurses. Japan Journal of Critical Care Nursing, 8, 134-147.

[11] Kabat-Zinn, J. (1990). Full catastrophe living: using the wisdom of your body and mind to face stress, pain and illness. Delacourt, New York.

[12] Speca, M., Carlson, L.E., Goodey, E. and Angen, M. (2000). A randomized, wait-list controlled clinical trial: The effect of a mindfulness meditation-based stress reduction program on mood and symptoms of stress in cancer outpatients. Psychosomatic Medicine, 62, 613622.

[13] Carlson, L.E., Speca, M., Patel, K.D. and Goodey, E. (2004). Mindfulness-based stress reduction in relation to quality of life, mood, symptoms of stress and levels of cortisol, dehydroepiandrosterone sulfate (DHEAS) and 
melatonin in breast and prostate cancer outpatients. Psychoneuroendocrinology, 29, 448-474. doi:10.1016/S0306-4530(03)00054-4

[14] Monti, D.A., Peterson, C. and Kunkel, S. (2006). A randomized, controlled trial of mindfulness-based art therapy (MBAT) for women with cancer. Psycho-Oncology, 15, 363-373. doi:10.1002/pon.988

[15] Garland, S.N., Carson, L.E., Cook, S., Lansdell, L. and Speca, M. (2007). A non-randomized comparison of mindfulness-based stress reduction and healing arts programs for facilitating post-traumatic growth and spirituality in cancer outpatients. Supportive Care in Cancer, 15, 949-961. doi:10.1007/s00520-007-0280-5

[16] Yamazaki, Y. and Yoshii, K., Trans. (2001). Unraveling the mystery of health: How people manage stress and stay well (in Japanese). Yushindokomunsya, Japan.

[17] Nakagawa, T. and Taibou, I. (1985). Japanese version of the GHQ mental health inventory handbook. Nihon Bunka Kagakusya, Japan.

[18] Funashima, N. (2001). Qualitative analysis (in Japanese). Igakusyoin, Japan.

[19] Tacon, A.M., Caldera, Y.M. and Ronaghan, C. (2004). Mindfulness-based stress reduction in women with breast cancer. Families Systems Health, 22, 193-203. doi:10.1037/1091-7527.22.2.193

[20] Tacon, A.M., Caldera, Y.M. and Ronaghan, C. (2005). Mindfulness, psychosocial factors, and breast cancer. Journal of Cancer Pain and Symptom Palliation, 1, 4553. doi:10.1300/J427v01n01_06

[21] Shimizu, T., Kubota, S., Mishima, T. and Nagata, S. (2004). Relationships between self-esteem and assertiveness training among Japanese hospital nurses. Journal of Occupational Health, 46, 296-298. doi:10.1539/joh.46.296 